\title{
Loss of podocalyxin causes a novel syndromic type of congenital nephrotic syndrome
}

\author{
Hee Gyung Kang1,2,7, Moses Lee ${ }^{3,7}$, Kyoung Boon Lee ${ }^{4}$, Michael Hughes ${ }^{5}$, Bo Sang Kwon ${ }^{1}$, Sangmoon Lee ${ }^{3}$, \\ Kelly M McNagny ${ }^{5}$, Yo Han Ahn ${ }^{1,2}$, Jung Min Ko ${ }^{1,2}$, Il-Soo $\mathrm{Ha}^{1,6}$, Murim Choi ${ }^{1,3,6}$ and Hae Il Cheong ${ }^{1,2,6}$ \\ Many cellular structures directly imply specific biological functions. For example, normal slit diaphragm structures that extend \\ from podocyte foot processes ensure the filtering function of renal glomeruli. These slits are covered by a number of surface \\ proteins, such as nephrin, podocin, podocalyxin and CD2AP. Here we report a human patient presenting with congenital \\ nephrotic syndrome, omphalocele and microcoria due to two loss-of-function mutations in PODXL, which encodes podocalyxin, \\ inherited from each parent. This set of symptoms strikingly mimics previously reported mouse Podx/ ${ }^{-/}$embryos, emphasizing the \\ essential function of PODXL in mammalian kidney development and highlighting this patient as a human PODXL-null model. \\ The results underscore the utility of current genomics approaches to provide insights into the genetic mechanisms of human \\ disease traits through molecular diagnosis.
}

Experimental \& Molecular Medicine (2017) 49, e414; doi:10.1038/emm.2017.227; published online 15 December 2017

\section{INTRODUCTION}

The majority of congenital nephrotic syndrome (CNS) or infantile nephrotic syndrome causative genes, such as nephrin, ${ }^{1,2}$ podocin, ${ }^{2,3}$ the transcription factor $W T 1,{ }^{2,4}$ and the glomerular basement membrane matrix component laminin, ${ }^{5-7}$ encode essential components of glomerular epithelial cells or podocytes. Extrarenal symptoms often accompany CNS. Examples include microcoria in Pierson syndrome, which is characterized by LAMB2 defects, ${ }^{5-7}$ and ambiguous genitalia or a diaphragmatic defect in patients with WT1 mutations. $^{4}$

CD34-family sialomucins, including CD34, podocalyxin-like 1 (PODXL) and endoglycan (PODXL2), comprise a small family of cell surface glycoproteins that confer the unique functions of blocking adhesion and enhancing mobility. The functions of these proteins are potentially mediated by the shielding of integrins and other adhesion molecules with glycosylated, highly charged mucin domains. ${ }^{8}$ Although sharing common expression domains, each member has specific roles and displays unique phenotypes when deleted in mice. For example, $P o d x l$ is uniquely expressed by kidney podocytes and embryonic mesothelial cells, and Podxl ${ }^{-1-}$ mice die perinatally due to defects in these cell types. ${ }^{9}$ Nevertheless, direct evidence of a PODXL requirement during human development remains elusive. $^{10}$

Recent genome scanning efforts have generated numerous individual-level genome sequences and enabled so called 'human knockout projects', which allows for the screening of genes that are functionally inactive in healthy individuals. ${ }^{11,12}$ Likewise, pairing an individual with specific clinical presentations of rare loss-of-function (LoF) mutations in a specific gene provides valuable insights into gene function and disease pathogenesis. For example, individuals who lack the leptin hormone become extremely obese during early life, phenocopying mouse models. ${ }^{13}$ Here we report the discovery and phenotypic analysis of a PODXL-null individual suffering from CNS and other defects that almost completely phenocopy Podxl knockout mice. ${ }^{9}$

\footnotetext{
${ }^{1}$ Department of Pediatrics, Seoul National University College of Medicine, Seoul, Republic of Korea; ${ }^{2}$ Research Coordination Center for Rare Diseases, Seoul National University Hospital, Seoul, Republic of Korea; ${ }^{3}$ Department of Biomedical Sciences, Seoul National University College of Medicine, Seoul, Republic of Korea; ${ }^{4}$ Department of Pathology, Seoul National University College of Medicine, Seoul, Republic of Korea; ${ }^{5}$ The Biomedical Research Centre, The University of British Colombia, Vancouver, British Columbia, Canada and ${ }^{6}$ Kidney Research Institute, Medical Research Center, Seoul National University College of Medicine, Seoul, Republic of Korea

${ }^{7}$ These authors contributed equally to this work.

Correspondence: Professor M Choi, Department of Biomedical Sciences, Seoul National University College of Medicine, 103 Daehang-no, Jongno-Gu, Seoul 03080, Republic of Korea.

E-mail: murimchoi@snu.ac.kr

or Professor HI Cheong, Department of Pediatrics, Seoul National University College of Medicine, 101 Daehang-no, Jongno-Gu, Seoul 03080, Republic of Korea.

E-mail: cheonghi@snu.ac.kr

Received 13 March 2017; revised 1 July 2017; accepted 11 July 2017
} 


\section{MATERIALS AND METHODS}

\section{Study approval}

All patient-related procedures were approved by the Seoul National University Hospital Institutional review board, approval No. 0812002-264.

\section{Whole exome sequencing and variant calling}

The procedures used for the preparation of genomic DNA, whole exome capture using an Agilent V5 array, sequencing using an Illumina HiSeq 2500 platform (San Diego, CA, USA), read alignment, variant calling, variant filtering and de novo variant calling have been described previously. ${ }^{14}$

\section{Sanger sequencing}

PCR amplification was performed with 10 pmol of each specific primer, as listed in Supplementary Table S2. The PCR conditions were an initial denaturation at $95^{\circ} \mathrm{C}$ for $3 \mathrm{~min}$, followed by 35 cycles of amplification $\left(95^{\circ} \mathrm{C}\right.$ for $30 \mathrm{~s}, 60^{\circ} \mathrm{C}$ for $30 \mathrm{~s}$ and $72^{\circ} \mathrm{C}$ for $\left.30 \mathrm{~s}\right)$ and a final extension at $72{ }^{\circ} \mathrm{C}$ for $5 \mathrm{~min}$. The PCR product was gel extracted and Sanger sequenced on an ABI3730XL DNA Analyzer instrument (Applied Biosystems, Foster City, CA, USA).

\section{Immunohistochemistry}

The expression of PODXL was assessed by immunohistochemistry staining with a polyclonal goat antibody against human PODXL (IgG, Cat.\# AF1658, R\&D systems, Minneapolis, MN, USA, 1:100 dilution). A $4 \mu \mathrm{m}$ section of the patient's omphalocele tissue was subjected to antigen retrieval using a cloak chamber in $\mathrm{pH} 6.0$ citrate buffer at $100^{\circ}$ $\mathrm{C}$ for $1 \mathrm{~h}$. Subsequently, the tissue was stained using an UltraVision LP kit (Lab Vision corporation, Fremont, CA, USA) and a Bond Polymer Refine Detection kit (Leica, Wetzlar, Germany). A tissue microarray composed of 59 various normal human and cancer tissues were used as a control (Superbiochips, Seoul, Korea).

\section{Cell culture, transfection and western blotting}

HEK293 cells and HeLa cells were cultured in high-glucose DMEM media with $1 \%$ antibiotics. The media was changed to high-glucose DMEM without antibiotics for 1 day before transfection. The GeneINTM transfection reagent GST-1002 was used to transfect cells with wild-type, M1I and W341X PODXL cDNAs cloned into a pcDNA3.1+ vector. Forty-eight hours after transfection, the cells were lysed with RIPA buffer, separated on an $8 \%$ SDS-PAGE gel, transferred, and probed using antibodies diluted in 5\% skim milk. The antibodies used were goat anti-PODXL (R\&D, AF1658, 1:2500), mouse anti- $\alpha$-tubulin (Santa Cruz, sc-8035, 1:20 000), rabbit anti-goat IgG conjugated to HRP (Abcam, Cambridge, UK, ab6741, 1:5000), and goat anti-mouse IgG conjugated to HRP (Bio-Rad, Hercules, CA, USA, $170-6516,1: 5000)$.

\section{qPCR analysis}

qPCR was performed with a cDNA library from transfected HeLa cells, according to the manufacturer's instructions (Bioneer AccuPower RocketScript RT PreMix, Alameda, CA, USA). The primers for PODXL were $5^{\prime}$-AACCCGGCCCAAGATAAGTG- $3^{\prime}$ and 5'-GGCAGGGAGCTTAGTGTGAA-3', forward and reverse, respectively. The reverse primer for $P O D X L$ was designed to span exon6-exon7. The primer sequences for $G A P D H$ were 5'-ACAACTTTGGTATCGTGGAAGG-3' and 5'-GCCATCACGCCA CAGTTTC-3', forward and reverse, respectively. The primer for GAPDH was designed to span exon1-exon7.

\section{RESULTS}

A patient with unknown congenital nephrotic syndrome

A newborn male infant was admitted to the neonatal intensive care unit for prematurity and congenital omphalocele. The infant was born as a preterm spontaneous delivery at a gestational age (GA) of 36 weeks and a bodyweight of $2.81 \mathrm{~kg}$. The placenta was large, with a weight of $800 \mathrm{~g}$. The child was conceived via in vitro fertilization and embryo transfer (IVF-ET) and carried by a 37-year-old mother with Turner syndrome $(45, \mathrm{X} / 46, \mathrm{XX})$ mosaicism. Congenital omphalocele was diagnosed by prenatal screening ultrasonography at GA 22 weeks. A previous pregnancy by the infant's parents, also through an IVF-ET, produced an in utero fetal death at GA 38 weeks. Other family history was denied. On physical examination, the newborn presented with omphalocele (Figure 1a), a webbed neck, bilateral simian creases, a flat round face, flat nasal bridge, up-slanting palpebral fissures, epicanthal folds and microcoria. The laboratory tests after birth revealed a serum albumin concentration of $2.6 \mathrm{~g} \mathrm{dl}^{-1}$ and proteinuria $3+$, with microscopic hematuria on urinalysis. The initial serum creatinine (Scr) levels were $0.41 \mathrm{mg} \mathrm{dl}^{-1}$ (Figure 1b). The kidneys were relatively large, with increased cortical echogenicity and poor corticomedullary differentiation on ultrasonography (Figure 1c). After primary repair of the omphalocele on day 3, the patient developed aggravated hypoalbuminemia and Scr up to $1.3 \mathrm{mg} \mathrm{dl}^{-1}$, with a gradual decrease in urine output (Figure 1b). On day 18, severe pulmonary hypertension with right to left shunt through the patent ductus arteriosus was noted and managed with sildenafil, nitric oxide inhalation and mechanical ventilator care. Generalized edema became evident despite regular albumin replacement by 2 weeks of age. On day 22, continuous renal replacement therapy was applied and later switched to peritoneal dialysis. Thyroid hormone and immunoglobulin were replaced regularly; however, the patient's feeding was poor, and he suffered from repeated infections, including pneumonia, central catheter infection by Acinetobactor baumanii and subsequent peritoneal dialysis-related peritonitis by Stenotrophomonas maltophilia and Candida parapsilosis (Figure 1b). On day 48, seizures developed without an identifiable cause, and his mental status deteriorated. The patient experienced repeated septic shock events as well as brain atrophy (Figure 1d), ventriculomegaly, and encephalomalacia. The patient expired due to sepsis at the age of 130 days.

\section{PODXL is mutated in the patient}

The presentation of microcoria with CNS led us to suspect Pierson syndrome, ${ }^{5-7}$ but targeted screening of $L A M B 2$, a known culprit of Pierson syndrome, did not reveal a pathogenic variant. We next sought the genetic cause of the patient's defects using a trio-based whole exome sequencing (WES) approach (Supplementary Table 1). Out of 45,676 variants called, the base-read quality score and population-based filtering using the 1000 Genomes, Exome Aggregation Consortium (ExAC) $)^{15}$ and Korean in-house databases as references led to the identification of 149 rare variants. Among these 

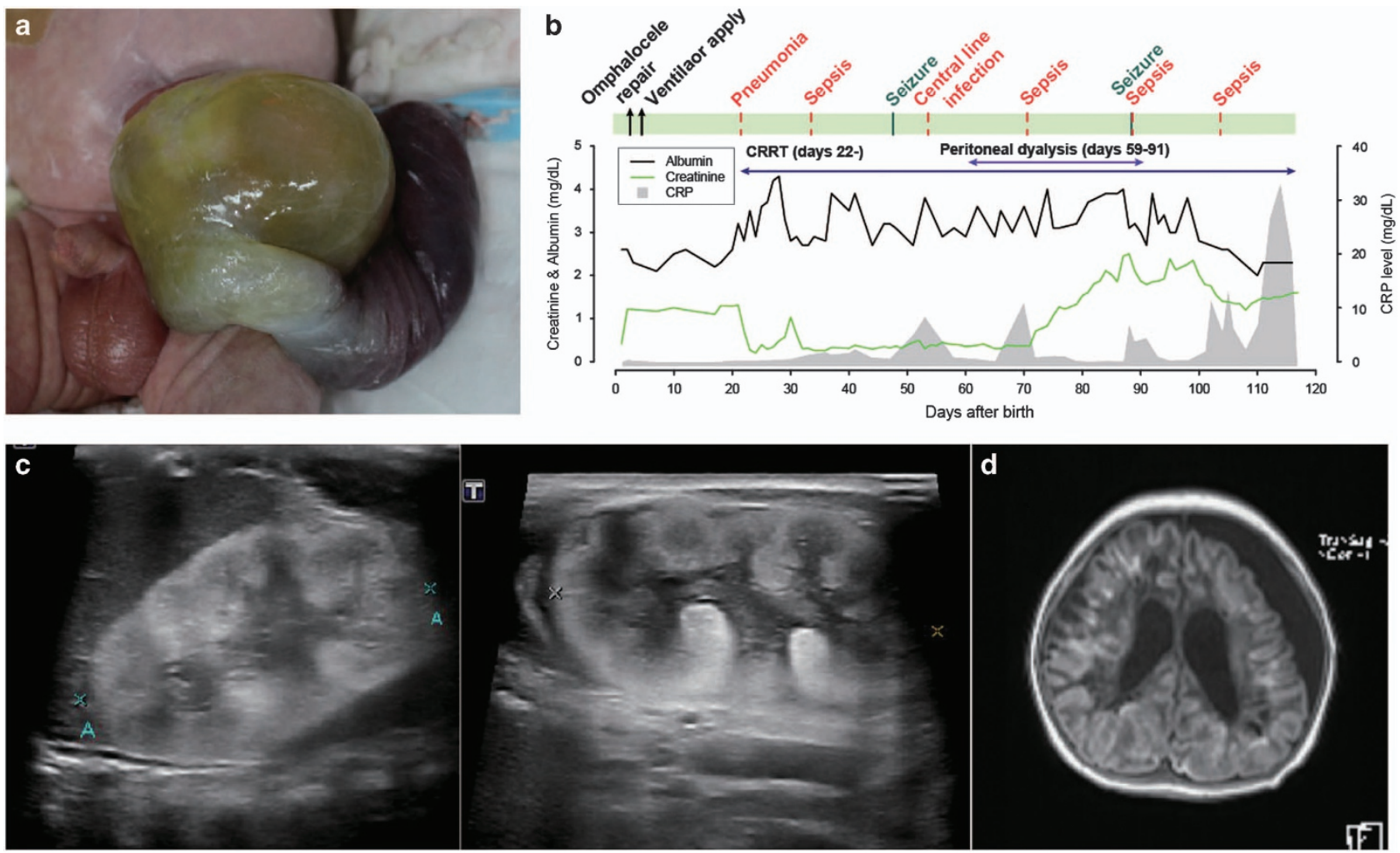

Figure 1 Clinical findings from a case with a defective PODXL gene. (a) Omphalocele. (b) Clinical course with recurrent infection episodes. (c) Ultrasonography of the kidneys with increased echogenicity. (d) Brain MRI with enlarged ventricles.

variants, three genes with potentially disease-causing variants were identified, either in the form of de novo or compound heterozygous variants (Supplementary Table 2). No notable structural variation was identified (Supplementary Figure 1). The patient sample was also analyzed by $\sim 47 \times$ coverage whole-genome sequencing and was found to be negative for balanced translocations (data not shown). The variants in PODXL (podocalyxin like) were a missense mutation at the initiation codon c.3G $>\mathrm{T}$ (p.Met1Ile) and a nonsense mutation at c.1023G $>A$ (p.Trp341Ter), inherited from the father and the mother, respectively (Figure 2a). The next ATG initiation sequence was found at the c.98 position in a different frame, which would generate a truncated protein of 31 amino acids. Both of the variants were validated by Sanger sequencing (Figure 2b, Supplementary Table 3) and are not listed in the public databases or an in-house database of 1060 healthy Koreans, as described above. As the two variants are likely to cause a LoF protein, we searched for the existence of individuals carrying potential PODXL LoF variants. From the ExAC database, ${ }^{15} 22$ out of 120000 grossly healthy individuals' chromosomes harbor one LoF variant in PODXL, resulting in a very low likelihood of finding individuals carrying two damaged alleles (Supplementary Table 4, $P=3.4 \times 10^{-8}$ ). Furthermore, our in-house exome database of 1020 healthy Koreans did not reveal any individual with more than one damaging variant in PODXL. Both observations support that the gene is intolerable to homozygous LoF changes in healthy individuals. The two other candidate genes, FUT1 and CPEB2, were ruled out as less relevant based on previous disease associations and expression patterns (Supplementary Table 2, Supplementary Figures 2 and 3).

\section{PODXL protein is ablated in the patient tissue}

To determine whether the two presumed LoF variants in the patient inhibit the production of functional proteins, we introduced these variants in a PODXL expression construct and assayed mutant protein expression in HEK293 (Figure 2c) and HeLa cells (Supplementary Figure 4). As expected, and unlike the wild-type PODXL, both PODXL mutant constructs failed to induce the expression of PODXL protein. Because patient kidney tissue was unavailable for biochemical analyses, we performed an immunohistochemical evaluation of the patient's omphalocele tissue (containing small vascular channels), along with the omphalocele tissues from two individuals with umbilical hernia (Figure 2d-i, Supplementary Figure 5). PODXL is known to be expressed by virtually all vascular endothelial cells in mouse and human.9,16,17 Therefore, its presence or absence in the patient could be evaluated in endothelial cells of the patient's omphalocele tissue. Both patient and control endothelial cells were positively stained for endothelial marker CD34, as expected (Figure 2e and h), but PODXL expression was undetectable in the patient tissue (Figure $2 \mathrm{f}$ and $\mathrm{i}$ ). Omphalocele tissues consist of varying proportions of fibrous tissues with microvessels (Figure 2d and g). Although the histological stains appeared different between the patient and control tissues, with the control tissue 
a

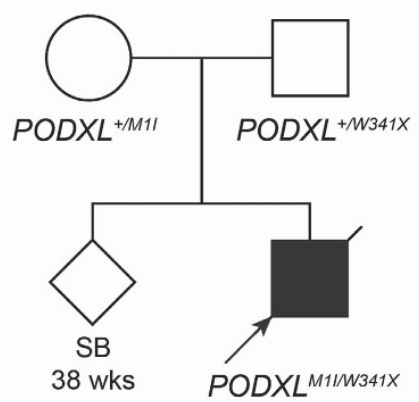

b

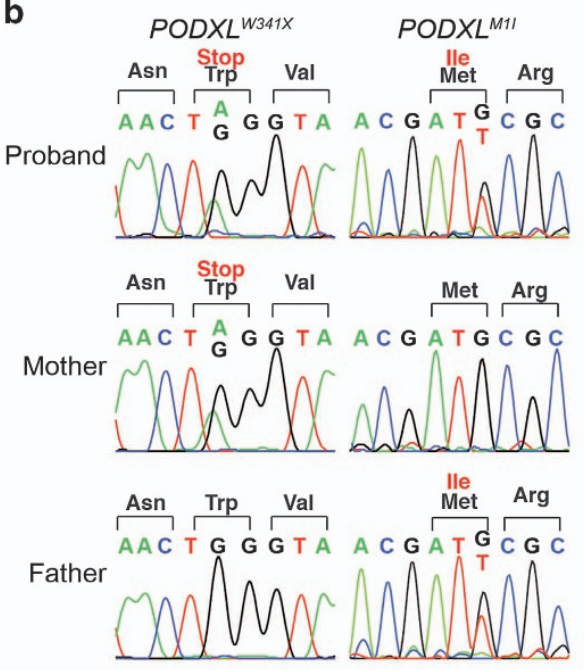

C
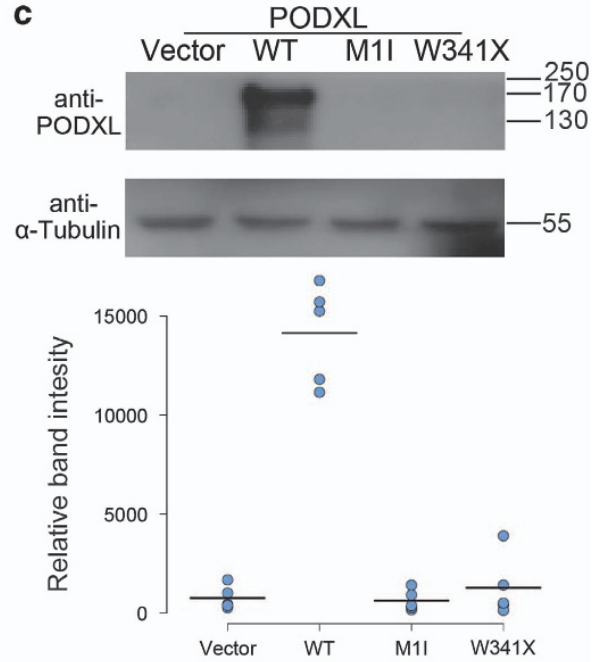
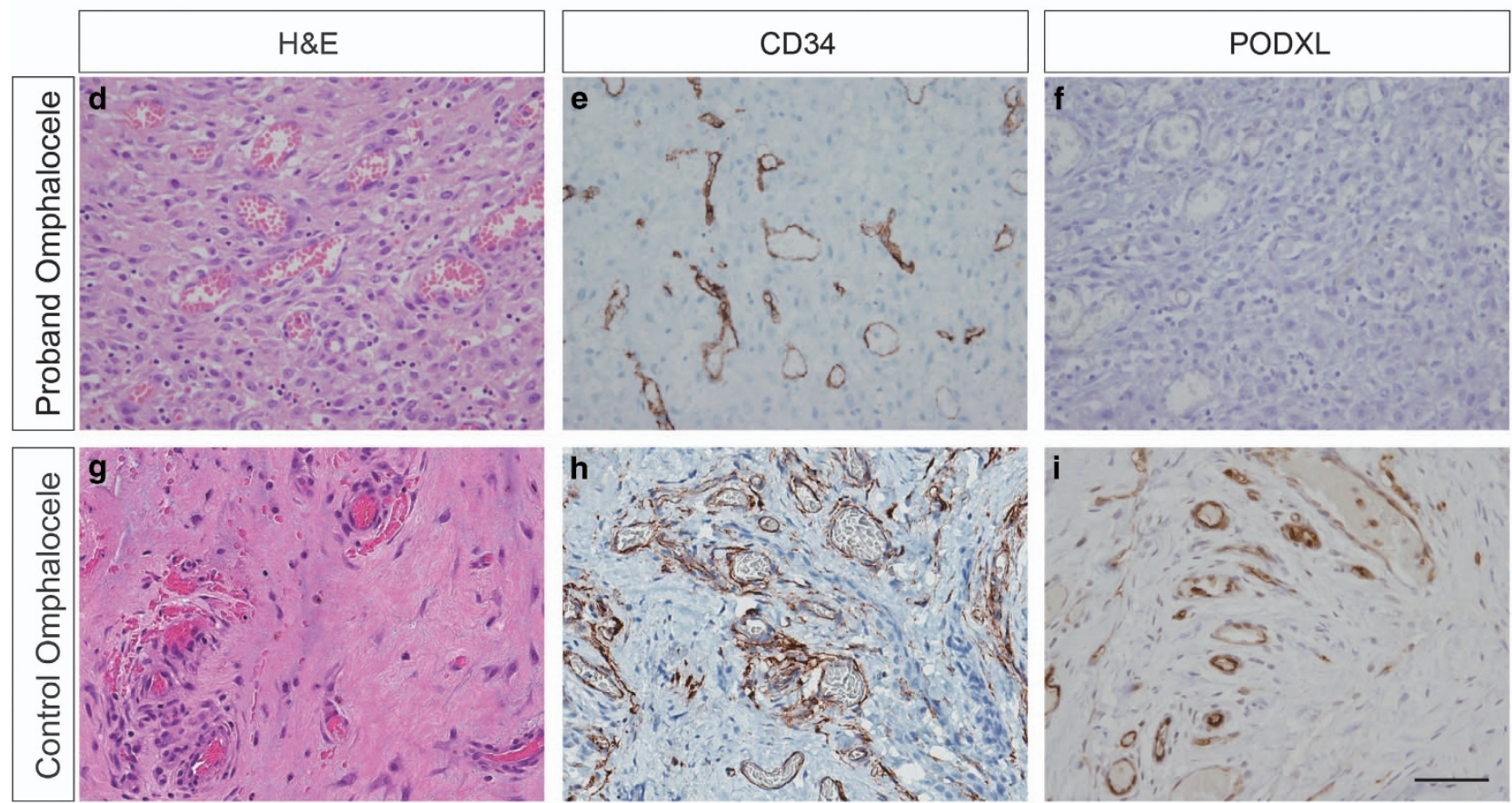

Figure 2 Loss-of-function PODXL variants do not produce a functional protein. (a) Pedigree of a family with congenital nephrotic syndrome. The proband had compound heterozygous variants in PODXL, with c.3G $>$ T (p.Met1lle) from the healthy father and c.1022G $>A$ ( $p$. Trp341X) from the healthy mother. (b) Sanger sequencing traces confirming the variant calls. (c) Western blot of wild-type or mutant PODXL expressed in HEK293 cells. Five replicates resulted in almost complete loss of podocalyxin expression following transfection with either of the mutants. (d-i) Immunohistochemistry of the patient's omphalocele tissue, demonstrating a complete lack of PODXL expression. (d-f) Omphalocele tissue from an unaffected control individual (g-i). (d, g) H\&E staining. (e, h) CD34 staining for endothelial cells. (f, i) PODXL staining (original magnification $\times 400$ ).

displaying denser collagenous stroma, this difference seems to be within the normal morphological variance of omphalocele tissues and is unlikely to be due to the PODXL mutations.

\section{DISCUSSION}

PODXL is a heavily charged transmembrane sialomucin with a weight of $140-165 \mathrm{kD} .^{16,18}$ This protein is abundantly expressed on the apical cell surface of podocytes, vascular endothelial cells, mesothelial cells lining the coelomic cavity, hematopoietic precursor cells and the developing brain., ${ }^{9,16,17,19,20}$ PODXL contains a highly charged cytoplasmic tail that contains phosphorylation sites for protein kinase $\mathrm{C}$ as well as an ezrin-binding domain through which PODXL attaches to an actin cytoskeleton. ${ }^{21}$ As PODXL is the predominant glycocalyx protein on podocytes, the anionic charge of this molecule has been considered to function as a charge barrier in glomerular filtration and to play a charge repulsion role that maintains the space between the podocyte interdigitating foot processes (FPs). ${ }^{22,23}$ Therefore, it has been postulated that loss or mutation of this protein may cause nephrotic syndrome or renal failure. This 
hypothesis is also partially supported by the report that a PODXL variant may cause familial focal segmental glomerulosclerosis. ${ }^{24}$ Direct supporting evidence for this hypothesis comes from our previous study demonstrating that the disruption of Podxl in mice results in a loss of FPs in podocytes, anuric renal failure and perinatal mortality. The conserved function of human and mouse Podxl in podocytes is further supported by a recent in vitro analysis of PODXL-null human iPSC-derived podocytes. ${ }^{25}$ Finally, the negative charge of Podxl is also thought to provide an anti-adhesive surface during retraction of the gut through umbilical ring, and $\sim 30 \%$ of the Pod $x l^{-/-}$pups display omphalocele. ${ }^{9}$ These results provide compelling evidence that PODXL LoF variants caused CNS with omphalocele in this patient.

During the preparation of this manuscript, a study reporting PODXL LoF mutations in a family with juvenile Parkinsonism but apparently normal kidneys was published. ${ }^{26}$ While the link to Parkinsonism is intriguing given PODXL expression in hippocampal neurons, we noted in a follow-up report that the 'null' phenotype in these patients was based on weak genetic data from a GC-rich sequencer that likely detected a more common in-frame variant allele. ${ }^{27}$ The data described here are more likely to document a true, germ line null allele in the PODXL gene in humans, and this conclusion is further supported by the striking similarities observed between this patient and Podxl KO mice.

Sanger sequencing and WES both failed to reveal any potentially pathogenic variants in $L A M B 2$ in our patient (Supplementary Figure 6). The cause of microcoria in our patient or in Pierson syndrome remains unclear. Although we evaluated $P o d x l^{-1-}$ pups for a similar defect, the wild-type and mutant animals displayed comparable pupil sizes (Supplementary Figure 7).

Pulmonary hypertension aggravated the patient condition. It is noteworthy that mice with a vascular endothelial-specific PODXL deletion exhibit increased lung volume, altered matrix composition, and increased pulmonary vascular permeability, ${ }^{28}$ implying a role for endothelial PODXL in pulmonary hypertension. Further analyses to reveal the pulmonary mechanism of hypertension pathogenesis in humans lacking functional PODXL are required.

The patient described here succumbed to recurrent severe infections with neurologic defects, which were considered to be sequelae of sepsis. However, a direct functional role for PODXL in the brain cannot be ruled out given that PODXL is widely expressed in the developing brain and the blood-brain barrier. ${ }^{16,20,29}$ Pan-neural ablation of this protein has been reported to result in ventricular enlargement, and LoF reduced the number of synapses in the brain. ${ }^{16,30}$ Thus, a possible functional role for PODXL during brain developmental processes should be studied further.

In conclusion, we report the identification of another causative gene of human CNS, adding an additional layer of complexity to CNS pathophysiology. This is the first described case of a PODXL-null individual who phenocopied a constellation of developmental defects displayed by mice that lack the gene (Supplementary Table 5). The results provide a further example of a human knockout model that supports what was previously known about protein functions from the study of animal model systems. Notably, and adding to the importance of this case, these similarities held in several biological contexts.

\section{CONFLICT OF INTEREST}

The authors declare no conflict of interest.

\section{ACKNOWLEDGEMENTS}

We thank the patient and family members for their participation in the study and Dr H Gee for his critical reading of the manuscript. This work was supported by grants from the Korean Health Technology R\&D Project, Ministry of Health and Welfare (HI12C0014), and the National Research Foundation, which is funded by the Ministry of Science, ICT and Future Planning (NRF-2014R1A1A2A16053266).

\section{PUBLISHER'S NOTE}

Springer Nature remains neutral with regard to jurisdictional claims in published maps and institutional affiliations.

1 Kestila M, Lenkkeri U, Mannikko M, Lamerdin J, McCready P, Putaala H et al. Positionally cloned gene for a novel glomerular protein-nephrin-is mutated in congenital nephrotic syndrome. Mol Cell 1998; 1: 575-582.

2 Santin S, Bullich G, Tazon-Vega B, Garcia-Maset R, Gimenez I, Silva I et al. Clinical utility of genetic testing in children and adults with steroid-resistant nephrotic syndrome. Clin J Am Soc Nephrol 2011; 6: 1139-1148.

3 Boute N, Gribouval O, Roselli S, Benessy F, Lee H, Fuchshuber A et al. NPHS2, encoding the glomerular protein podocin, is mutated in autosomal recessive steroid-resistant nephrotic syndrome. Nat Genet 2000; 24: 349-354.

4 Schumacher V, Scharer K, Wuhl E, Altrogge H, Bonzel KE, Guschmann M et al. Spectrum of early onset nephrotic syndrome associated with WT1 missense mutations. Kidney Int 1998; 53: 1594-1600.

5 Zenker M, Aigner T, Wendler O, Tralau T, Muntefering H, Fenski R et al. Human laminin beta2 deficiency causes congenital nephrosis with mesangial sclerosis and distinct eye abnormalities. Hum Mol Genet 2004; 13 : 2625-2632.

6 Hasselbacher K, Wiggins RC, Matejas V, Hinkes BG, Mucha B, Hoskins BE et al. Recessive missense mutations in LAMB2 expand the clinical spectrum of LAMB2-associated disorders. Kidney Int 2006; 70: 1008-1012.

7 Choi HJ, Lee BH, Kang JH, Jeong HJ, Moon KC, Ha IS et al. Variable phenotype of Pierson syndrome. Pediatr Nephrol 2008; 23: 995-1000.

8 Nielsen JS, McNagny KM. Novel functions of the CD34 family. J Cell Sci 2008; 121(Pt 22): 3683-3692.

9 Doyonnas R, Kershaw DB, Duhme C, Merkens H, Chelliah S, Graf T et al. Anuria, omphalocele, and perinatal lethality in mice lacking the CD34-related protein podocalyxin. J Exp Med 2001; 194: 13-27.

10 Nielsen JS, McNagny KM. The role of podocalyxin in health and disease. J Am Soc Nephrol 2009; 20: 1669-1676.

11 Alsalem AB, Halees AS, Anazi S, Alshamekh S, Alkuraya FS. Autozygome sequencing expands the horizon of human knockout research and provides novel insights into human phenotypic variation. PLoS Genet. 2013; 9: e1004030.

12 Sulem $P$, Helgason $H$, Oddson A, Stefansson H, Gudjonsson SA, Zink F et al. Identification of a large set of rare complete human knockouts. Nat Genet 2015; 47: 448-452.

13 Wabitsch M, Funcke JB, Lennerz B, Kuhnle-Krahl U, Lahr G, Debatin KM et al. Biologically inactive leptin and early-onset extreme obesity. $N$ Engl J Med 2015; 372: 48-54.

14 Seo J, Choi IH, Lee JS, Yoo Y, Kim NK, Choi M et al. Rare cases of congenital arthrogryposis multiplex caused by novel recurrent CHRNG mutations. J Hum Genet 2015; 60: 213-215. 
15 Lek M, Karczewski KJ, Minikel EV, Samocha KE, Banks E, Fennell T et al. Analysis of protein-coding genetic variation in 60,706 humans. Nature 2016; 536: 285-291.

16 Vitureira N, Andres R, Perez-Martinez E, Martinez A, Bribian A, Blasi J et al. Podocalyxin is a novel polysialylated neural adhesion protein with multiple roles in neural development and synapse formation. PLOS ONE 2010; 5: e12003.

17 Kerjaschki D, Sharkey DJ, Farquhar MG. Identification and characterization of podocalyxin-the major sialoprotein of the renal glomerular epithelial cell. J Cell Biol 1984; 98: 1591-1596.

18 Horrillo A, Porras G, Ayuso MS, Gonzalez-Manchon C. Loss of endothelial barrier integrity in mice with conditional ablation of podocalyxin (PodxI) in endothelial cells. Eur J Cell Biol 2016; 95: 265-276.

19 Dekan G, Gabel C, Farquhar MG. Sulfate contributes to the negative charge of podocalyxin, the major sialoglycoprotein of the glomerular filtration slits. Proc Natl Acad Sci USA 1991; 88: 5398-5402.

20 Vitureira N, McNagny K, Soriano E, Burgaya F. Pattern of expression of the podocalyxin gene in the mouse brain during development. Gene Expr Patterns 2005; 5: 349-354.

21 Fukasawa H, Obayashi H, Schmieder S, Lee J, Ghosh P, Farquhar MG. Phosphorylation of podocalyxin (Ser415) prevents RhoA and ezrin activation and disrupts its interaction with the actin cytoskeleton. Am J Pathol $2011 ; 179$ : 2254-2265.

22 Schnabel E, Dekan G, Miettinen A, Farquhar MG. Biogenesis of podocaIyxin-the major glomerular sialoglycoprotein-in the newborn rat kidney. Eur J Cell Biol 1989; 48: 313-326.

23 Takeda T, Go WY, Orlando RA, Farquhar MG. Expression of podocalyxin inhibits cell-cell adhesion and modifies junctional properties in MadinDarby canine kidney cells. Mol Biol Cell 2000; 11: 3219-3232.

24 Barua M, Shieh E, Schlondorff J, Genovese G, Kaplan BS, Pollak MR. Exome sequencing and in vitro studies identified podocalyxin as a candidate gene for focal and segmental glomerulosclerosis. Kidney Int 2014; 85: 124-133.

25 Freedman BS, Brooks CR, Lam AQ, Fu H, Morizane R, Agrawal V et al. Modelling kidney disease with CRISPR-mutant kidney organoids derived from human pluripotent epiblast spheroids. Nat Commun 2015; 6: 8715.

26 Sudhaman S, Prasad K, Behari M, Muthane UB, Juyal RC, Thelma BK. Discovery of a frameshift mutation in podocalyxin-like (PODXL) gene, coding for a neural adhesion molecule, as causal for autosomal-recessive juvenile Parkinsonism. J Med Genet 2016; 53: 450-456.

27 McNagny KM, Refaeli I, Hughes MR, Lee M, Choi M. The putative role of Podxl in autosomal-recessive juvenile Parkinsonism. In: J Med Genet 2016; 53: 450-456.

28 Debruin EJ, Hughes MR, Sina C, Lu A, Cait J, Jian Z et al. Podocalyxin regulates murine lung vascular permeability by altering endothelial cell adhesion. PLOS ONE 2014; 9: e108881.

29 Agarwal N, Lippmann ES, Shusta EV. Identification and expression profiling of blood-brain barrier membrane proteins. J Neurochem 2010; 112: 625-635.

30 Nowakowski A, Alonso-Martin S, Gonzalez-Manchon C, Larrucea S, Fernandez D, Vilar $\mathrm{M}$ et al. Ventricular enlargement associated with the panneural ablation of the podocalyxin gene. Mol Cell Neurosci 2010; 43: 90-97.

(i) (-) $\odot$ This work is licensed under a Creative Commons Attribution-NonCommercial-NoDerivs $\quad \mathbf{4 . 0}$

International License. The images or other third party material in this article are included in the article's Creative Commons license, unless indicated otherwise in the credit line; if the material is not included under the Creative Commons license, users will need to obtain permission from the license holder to reproduce the material. To view a copy of this license, visit http://creativecommons.org/licenses/by-nc-nd/4.0/

(C) The Author(s) 2017

Supplementary Information accompanies the paper on Experimental \& Molecular Medicine website (http://www.nature.com/emm) 